\title{
EL ROBOT TAMBORILERO: SOBRE LA POSIBILIDAD DE UN ARTE COMPARTIDO CON LOS ROBOTS
}

\section{Iñigo Benito Molinero}

Universidad del País Vasco /Euskal Herriko Unibertsitatea. Dpto. Arte y Tecnología Doctorando

\section{Resumen}

A partir de una pequeña y performativa experimentación con uno de mis pequeños robots y usando el ruido como escusa expresiva este ensayo estudia en torno a la idea del robot en su transformación de objeto a sujeto. La práctica artística tecnológica que va desde el arte generativo de los 70 hasta las posiciones más políticas en el arte de los nuevos medios proponen el entorno perfecto para el ya de por si mezclado contexto del arte robótico. Una mirada creciente en aspectos sociales y políticos que da a los artistas la posibilidad de trabajar en la agencia, autonomía y creatividad de las propias creaciones tecnológicas. Tanto como para postularlas como seres políticos. Este texto es una corta ojeada a algunas de esas ideas y trabajos

Palabras clave: CREACIÓN; ROBOT; SUJETO; ARTE INTERACTIVO; AUTORÍA

\section{THE DRUMMER ROBOT: THE POSSIBILITY OF A SHARED ART WITH ROBOTS}

\section{Abstract}

Through a small performative experimentation with a little robot of mine and using noise as a recursive excuse, this essay works on the idea of the robot in its change from object to subject. Technologic artistic practices which go from generative art from the 70 s to political stances on new media art propose the perfect environment to work on the very mixed context of art and robotics. A increasing social and political way. That gives artists a possibility to work on the agency, autonomy and creativity of the technological creations themselves. So they can also become political beings. This text is a brief peek on some of such ideas and works.

Key words: CREATION; ROBOT; SUBJECT; INTERACTIVE ART; AUTHORSHIP

\footnotetext{
Benito Molinero, Iñigo. 2015. "El ruido del robot tamborilero: sobre la posibilidad de un arte compartido con la tecnología”. AusArt 3 (2): 143-152. DOI: 10.1387/ausart.15948
}

\section{AUSART}




\section{EL ROBOT TAMBORILERO}

De entre todos los robots con los que convivo, hay uno muy especial de $5 \mathrm{~cm}$ de alto, formato juguete, que funciona dándole cuerda. El robot, tiene apariencia de robot de los 80 , esa especie de antropomorfismo hecho con cajas y muchos botones y luces. Y "tecnológicamente" hablando es un robot tamborilero. Vaya que, si tuviese si tuviese un oficio o "Techne" este sería el de tocar el tambor.

Sin embargo, mi querido amigo de hojalata no toca el tambor. Al menos no tal y como nosotros entenderíamos que debería. Para empezar, su tambor no tiene una membrana percusionable que lo haría tambor, de modo que su instrumento es más bien una "caja-metálica-no-tambor", de hecho, todo su cuerpo es de hojalata, por lo tanto, el sonido que dicha percusión debiera hacer sería más bien un tintineo metálico. Para colmo, debido a ciertos problemas de movilidad, el robot nunca alcanza a golpear la caja con la "baqueta" de modo que el sonido ni siquiera llega a suceder como tal. Aunque carecemos de los detalles por parte del fabricante de su funcionamiento teórico, se aprecia a simple vista que un sonido limpio de percusión no estaba dentro de las prioridades de dicho diseño.

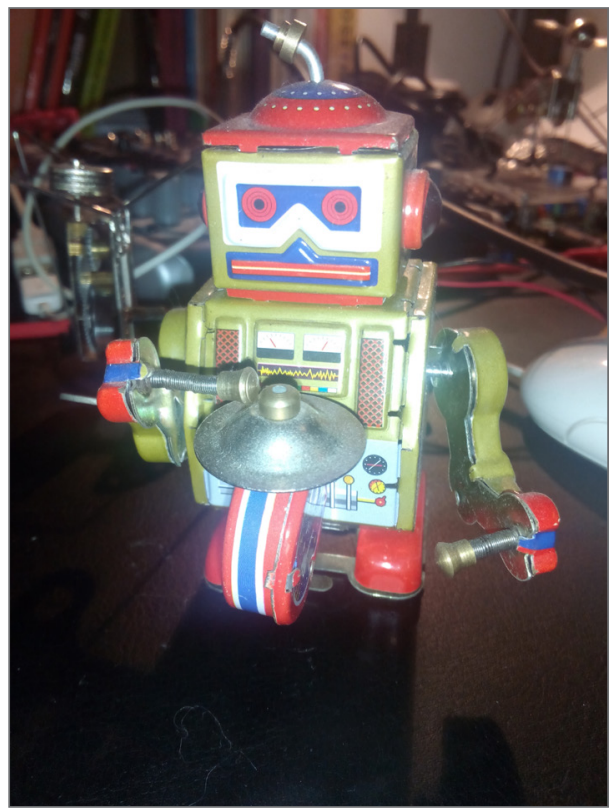

Fig. 1
Huelga decir que el robot SI hace ruido, vaya que sí. Desde que se gira la llave para darle cuerda, se puede apreciar la naturaleza sonora del juguete en cuestión, clac-clac-clac a cada medio giro de la rueda. Le sigue el zumbido interno del resorte que se libera, con el golpeteo de los pies contra el escritorio al caminar. Ambos sonidos desfallecen a medida que la energía se agota hasta que se detiene completamente. El único tambor que suena es el interno, el del resorte motor, y lo hace durante unos 10 segundos aproximadamente que dura toda la acción.

Decidido del potencial creativo de este objeto sonoro, preparo mi smartphone para grabarlo y prestar más atención. 
Durante esos instantes también hay más sonidos, menos perceptibles, más sutiles: mis dedos agarrando la llave, mi respiración, u otros ambientales, como el zumbido de una lámpara, o el aire que ulula en el micrófono.

Mi objetivo con esa microperformance es hacer de este robot un creador. ¿Es posible? La cadena creativa es desde luego inherente a los modos de fabricación y consumo postcapitalistas. Si bien yo, como creador, aporto unas dinámicas creativas y/o reflexivas para que "algo suceda", no he fabricado el robot, ni toco yo mismo el tambor, ni he programado el sensor de la cámara de mi smartphone que captura y hace visible la experiencia artística. La cadena creativa se sucede entre todas las partes del proceso. Como una suerte de cesión de la agencia que va asociada a lo que conforma el sujeto que es capaz de crear.

Cada una de las partes del proceso creativo, es indiscernible e indivisible de las demás, hasta tal punto que incluso en una experiencia tan pequeña el control sobre cada herramienta, tanto física como conceptual es imposible. Como mucho, tomamos decisiones concretas a fin de acercarnos a una propuesta. Si bien en las formas clásicas de arte la diferencia entre herramienta y autor parecía sencilla, la complejidad de la tecnología hace cada vez más difícil dicha situación, y para ello se necesitan nuevas líneas de pensamiento y trabajo. Cabría reflexionar, cuantitativa y cualitativamente si el trabajo creativo de esta o cualquier otra forma eminentemente tecnológica de arte, es efectuada "creativamente" por las herramientas que la conforman y no tanto por los auto proclamados autores de la misma.

\section{SOBRE CREACIÓN Y ARTES GENERATIVAS}

La primera, y más obvia, parada a la hora de reflexionar sobre esta idea de la autonomía tecnológica es en el arte generativo. Aunque se aceptan sus comienzos como anteriores a los 70 , el arte generativo y su impacto en la sociedad no se normalizó hasta la emergencia de la computación en los 90 y los 2000. Artistas de mil diversas disciplinas, desde las diferentes propuestas de la música algorítmica a las instalaciones interactivas con robots que reconocerían, no solo la creatividad de una máquina autónoma, sino la de un artista interesado en generar esas autonomías, y reconocer el interés político y social de tales creaciones. 
Una de las más fascinantes piezas de arte generativo de los 70 da pie a imaginar la capacidades de esa autonomía. John Horton Conway, matemático, propuso uno de los primeros juegos de cero jugadores (zero player games), es decir un juego cuya evolución está determinada por las condiciones iniciales y a partir de ahí funciona de forma autónoma. El denominado "Juego de la Vida", sugirió para muchos algo más que una serie de ciclos de computación y juegos matemáticos, en él se buscaba la posibilidad de un sistema con capacidad de generar más de lo imaginado a priori. Un juego en el borde del control. (fig.2)

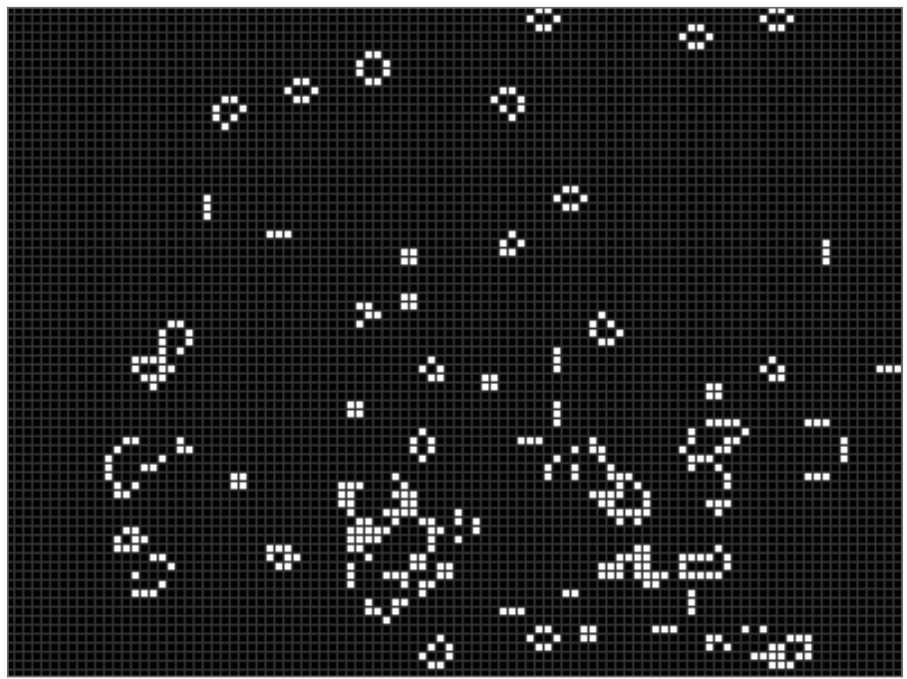

Fig. 2

Sin embargo, pese a ser temas detectados en el núcleo de la actividad creadora hace más de cuarenta años, las estructuras políticas de dicha relación con lo lógico y lo mecánico siguieron y siguen llenas de tabúes. Respecto a ciertos temas en la creación tecnológica, aun se perfilan problemáticas similares a la hora de agitar juicios y categorías. Estos están relacionados con la idea de que crear es un acto humano, un acto de inteligencia. Reconocer a ese "otro" como ente creativo, significaría compartir una capacidad que presumimos propia y única. Se trata en el fondo de la cuestión clásica del prometéo. Conformar piezas complejas de arte, o reflexiones a caballo entre lo crítico y lo poético no deja de ser nuestra actividad principal como creadores. Dar el fuego, ceder el concepto de la creatividad, nos resulta aun difícil. 
La capacidad para crear en sí no parece ser esclarecedora para estos temas, puesto que el progreso tecnológico es más rápido que su implementación social. Por decirlo de otra manera, mientras el potencial y la capacidad de las maquinas de producir se extiende y especializa, el creador humano comprende de forma cada vez menos pormenorizada la tecnología que usa. Continuando con el ejemplo inicial, a pesar de ser yo quien le da cuerda, es mi pequeño robot quién hace esos ruidos (sin que yo ni siquiera sepa exactamente cómo o qué ocurre en su interior). Por lo general, las maquinas son muy "capaces" de producir, generar o de crear, aunque solo sea acompañados por los humanos. Y aun así, la mayoría de seres humanos es reticente a considerar y mucho menos a valorar dicha colaboración.

Una de las características más extrañas de toda esta situación es que la capacidad generativa de dichos sistemas no depende de su capacidad perceptora. Para los seres humanos, la creación sucede tras un proceso de filtrado enorme de información, mientras que las creaciones de las máquinas suceden por un bombardeo o sobrecarga de cantidades de información no procesables. Están diseñadas para cosas tan concretas, que el resto del mundo es un contexto divergente para ellas. Cabría preguntarse qué tipo de estrategias podrían proponerse de cara a cuestionar el arte producido por robots. Y desde luego no son capacitativas, si acaso, todo lo contrario.

En "At One's Fingertips" de David Sanz Kirbis tenemos ese ejercicio de empatía necesaria para comprender la creación de ese "otro" programado, en este caso a partir de esa cuestión perceptiva. Se trata de una pieza audiovisual parte del proyecto Computer Visión Cinema: "un proyecto de investigación experimental para aplicar técnicas de visión por computadora en procesos cinematográficos, en la búsqueda de nuevos lenguajes expresivos audiovisuales" 1. La pieza está formada por dos visiones de un mismo acontecimiento, por un lado la información gráfica de los algoritmos de detección de una cámara, y la segunda una composición de dichas imágenes también grabadas por la cámara. Kirbis no sólo propone una aproximación a los procedimientos de la máquina sino a las estéticas de la misma, a su interés, a su mirada. Una mirada, frenética, inquieta, que en seguida muestra no estar asociada a la mirada humana, sino a otro tipo de condiciones. 


\section{DE OBJETO A SUJETO}

La segunda parada, pertenece a la necesidad inminente de actualizar el debate del sujeto y el objeto con respecto a la tecnología. Es un debate antiguo y complejo, pero de gran pertinencia, por lo que puntualizaré algunas cosas concretas para el caso que nos ocupa. Como decía Brea:

"El yo, desde luego, es una tecnología. Pero también los universos de la conciencia y la voluntad -soportan la mediación de una tecnología. La construcción lingüística del mundo de los artefactos, la ley que rige el sistema de los objetos, ¿cómo podría no proyectarse y determinar implacablemente la esfera de la conciencia -cuando en realidad ella es justamente la escritura que ésta, por su parte, dispone sobre el mundo real, objetivo?"

(Brea 2002, 135)

La oportunidad de repensar de nuevo la idea de sujeto está definitivamente asociada a la figura del robot. Y viene definida no solo por aquellas tecnologías que las conforman físicamente, sino también aquellas personales, sociales y relacionales con las que configuran y se unen al mundo. Esta división entre las denominadas tecnologías duras y las tecnologías blandas, no es estricta. Su relación es la clave para comprender cómo se conforma el sujeto contemporáneo.

Pero vayamos por partes. Para empezar sospecho que no es casualidad que el desarrollo de la robótica y la re-emergencia de textos y corrientes filosóficas en torno al sujeto y el objeto coincidan en el tiempo. Del mismo modo que no es casual que la producción cultural esté sufriendo un auge de reformulaciones de clásicos de ciencia ficción de diverso tipo. Entre el 2010 y el 2015 ha habido un aumento cualitativo de dichas expresiones. No es casual y no es inocente.

Mientras Ray Kurtzweil insiste en detectar las claves de la singularidad tecnológica, es a su vez contratado por google (2012) para desarrollar proyectos en aprendizaje automático y procesamiento de lenguaje natural dando pié a SIRI. Google a suvez realiza enormes inversiones alolargo de varios años absorbiendo empresas dedicadas a la robótica en diferentes campos. Las narrativas en torno a la robótica en el cine y la televisión entre 2010 y 2015 se suceden, se reafirman en los thrillers de corte dramático y/o apocalíptico propios de los discursos de la trascendencia cuasi metafísica. Mientras tanto tu roomba limpia bajo tu sofá. 
Tal complejidad, tantos acontecimientos tecnológicos (y muchos otros) simultáneos, sí son de crecimiento exponencial, tal y como los estudios de Kurtzweil demuestran, sin embargo no responden al quehacer político de los sujetos, sino que mi(s)tifican la convergencia de un millar de cuestiones en un momento, en una especie de momento extásico de la tecnología. De tal forma que pareciera que los transhumanistas y los posthumanistas hubieran sustituido una ascensión metafísica o religiosa por una tecnológica.

Mientras la problemática de la desaparición del autor sigue sin resolverse, sometida al bloqueo trascendental (Foucault [1969] 2010), la mediación en los discursos sobre robótica y tecnología es tal que resulta casi imposible dialogar sobre subjetividades en torno a ellas y a la creación, la condición posthumana se pelea por hacerse un hueco en ese impasse entre futurible e imaginada sin más resultados que una discusión y una latencia que se está estirando demasiado. En este sentido quizá deberíamos recordar las tres claves que Miguel Morey nos presenta en su introducción "la cuestión del método" para el libro de Foucault Tecnologías del yo:

“1) Evitar [...] universales antropológicos (y naturalmente también los de un humanismo que haga valer los derechos, los privilegios y la naturaleza de un ser humano como verdad inmediata e intemporal del sujeto.

2) Invertir el movimiento filosófico de ascenso hacia el sujeto constituyente [...] se trata por el contrario de descender al estudio de las prácticas concretas por las que el sujeto es constituido en la inmanencia [...].

3) Dirigirse como dominio de análisis a las "practicas", abordar el estudio por el sesgo de lo que "se hacía" [...]. Son las prácticas entendidas como un modo de actuar y a la vez de pensar las que dan la clave de inteligibilidad para la constitución correlativa del sujeto y del objeto."

(Morey Farré 1990).

Se trata, en consecuencia, de dejar espacio para que las tecnologías sucedan y comprender, desde la diferencia, la configuración de dicho sujeto tecnológico que no nos es ajeno, pero si alterno. 


\section{DISFUNCIÓN}

El tercer punto en el que nos detenemos para pensar en el robot como sujeto pertenece a la actualidad tecnológica y su uso. Las estéticas contemporáneas en torno a la tecnología están siempre en relación con lo interactivo, y muy a menudo con el fracaso del mismo. La disfunción, el mal funcionamiento, el error son parte del eje central de lo contemporáneo, y no lo es tanto por capricho estético. El vacío, lo negativo en aquello que está programado es lo que saca a relucir la naturaleza política de la tecnología.

Volviendo al ejemplo propuesto al inicio, y dado que el principal intento comunicativo de mi pequeño robot de hojalata es el musical, y éste no puede ser satisfecho debido a la disfunción de su brazo izquierdo (que es demasiado corto) dar cuerda a la maquina parece un ejercicio sensibilidad. No hacia el juguete (este no tiene ningún sensor y no siente) sino hacia el proceso creativo. Al ser activado el robot trabaja de forma insistente intentando golpear el tambor sin éxito, en una especie de estertor maquínico frustrante y agotador. Sin embargo el juguete no tiene otra forma de existir ni ninguna otra función, el ejercicio de hacerlo funcionar sin conseguir que funcione, o hacerlo funcionar mal si se quiere, hace que se pueda escuchar el proceso al que como objeto está atado. Al no poder sublimar su acción en el tambor, todo el resto del proceso se hace visible, o mejor dicho, escuchable.

También cabría pensar en la disfunción como un acto de rebeldía. El performer, robot de juguete en este caso, representa un sonido que nunca sucede mientras presenta el ruido de su cuerpo-objeto tal y como suena. Así, encontraríamos en la contradicción del acto, el debate sobre función y rendimiento, o si un robot parlantemente encendido está más satisfecho que uno permanentemente apagado. En cualquier caso, y hasta que se la indiscernibilidad entre agencia real y simulada sea posible, todo esto pertenece a otra discusión.

Quizá en el punto intermedio de ambos planteamientos, podemos extraer algunas ideas de las 000 (ontología orientada a objetos). Esta línea de pensamiento mantiene que los objetos existen independientemente de la percepción humana y por ende no agotan ontológicamente sus relaciones en base a las humanas. Este movimiento heredero de realismo especulativo nos interesa más metodológicamente que otra cosa, nos propone renunciar a ciertas categorías cuestionables de ordenar el mundo, aceptando otro tipo de relaciones 
diferentes a la sensibilidad y percepción humanas. Nos es útil por varias razones: Por un lado, el ejercicio especulativo es muy similar al de la ciencia ficción, la cual; a base de imaginar mundos posibles ha influenciado el desarrollo tecnológico mismo en gran medida. Y por otro, nos sitúa en un estado psicológico y social favorable para trabajar la aceptación de nuevas formas de ver y ordenar el mundo diferentes a las humanas. La disfunción, en este espectro ocurre entre las categorías sujeto y objeto, que se ven forzadas a una reformulación.

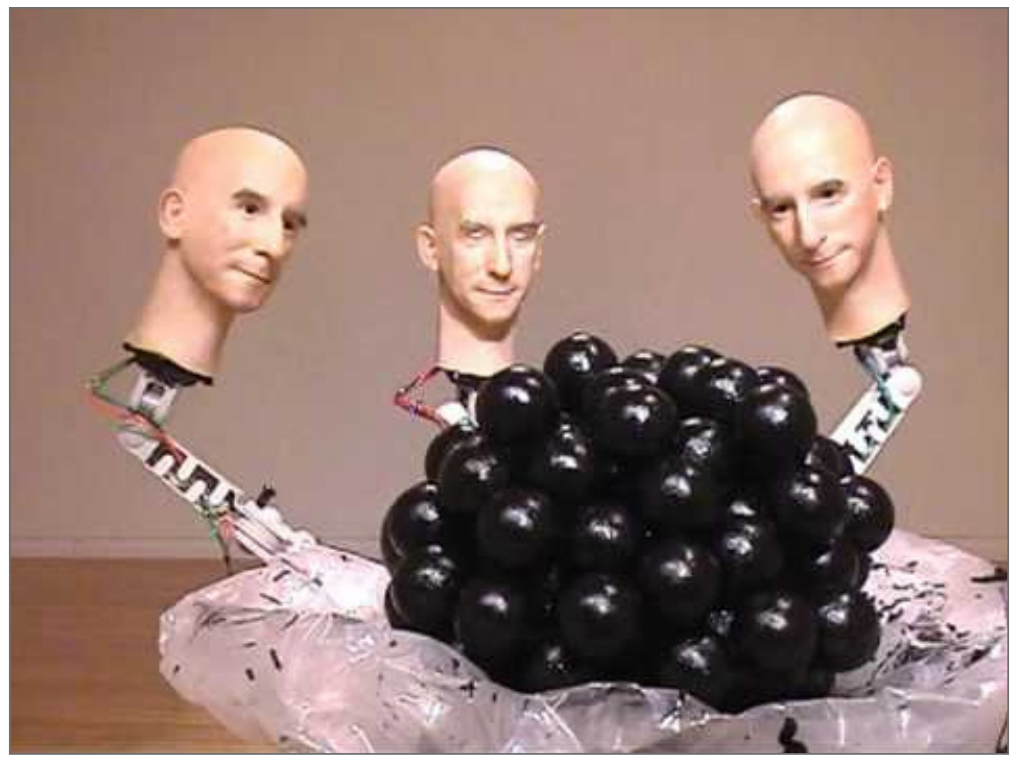

Fig. 3

Una pieza que abarca estas cuestiones es "The animal, vegetal and mineralness off everything" de Ken Fingold. Se trata de una instalación interactiva con forma de tres cabezas robóticas unidas a una plataforma que presenta un objeto. Las cabezas robóticas, cuyas caras son replicas en látex de la del propio artista, observan ese objeto tratando de adivinar si el objeto es animal, vegetal o mineral. Para ello las cabezas se hacen preguntas unas a otras y al público abriendo una especie de debate delirante. Es una obra que propone la opinión robótica como válida, pero también como propicia al debate y a la reflexión. Cada cabeza sostiene su subjetividad, al mismo tiempo que propone categorías en torno al objeto que tiene enfrente. Es un debate sin final, por momentos absurdo; pero no sin sentido, dado que cuestiona la naturaleza construida de los objetos y los sujetos, y de los límites de los mismos. La iro- 
nía que plantea Fingold, poniendo a los robots su propia cara, dándoles voz y diversidad, en última instancia convirtiéndolos en sujetos, hace que todo el debate entre en un ejercicio de metarobótica. Las cabezas por su parte, permanecen en un debate infinito limitadas tanto por su capacidad perceptiva como por la imposibilidad descriptiva de un lenguaje y unas categorías que ciertamente no pueden ser descritas de una única manera. (fig.3)

Esta doble visión tiene como propósito buscar al sujeto dentro del robot, una proyección de subjetividad sobre sustancia material, aun sabiendo que "no hay nadie en casa" (Zizek 2014, 92) pero igualmente deseándolo. Quizá incluso como una proposición: El sujeto robot un sujeto a nivel cero.

"Cuando uno mira a un sujeto autista a los ojos, uno también tiene la sensación de que no hay nadie en casa>>, pero, a diferencia de la presencia bruta de un objeto muerto como el cerebro, uno espera que haya algo/alguien ahí porque el espacio para ese alguien está ahí. Este es un sujeto a nivel cero, como una casa vacía en la que <<no hay nadie>>"

(Zizek 2014, 92).

\section{Referencias}

Brea, Jose Luis. 2002. La era postmedia. Salamanca: Centro de Arte de Salamanca

Foucault, Michel (1969) 2010. Qué es un autor? Traducción de Silvio Mattoni, seguido de Apostillas a "Qué es un autor?" por Daniel Link. Córdoba, Argentina: Ediciones Literales

- (1984). 1990. Tecnologías del yo y otros textos afines. Introducción de Miguel Morey, traducción de Mercedes Allende Salazar. Barcelona : Paidós

Morey Farré, Miguel. 1990. Introducción a Tecnologías del yo, de Michel Foucault, 9-43. BarceIona: Paidos

Sanz Kirbis, David. 2011. “At one's fingertips”, Vídeo de Vimeo, 7:00. Publicado 13 feb. 2012 https://vimeo.com/36708258

Zizek, Slavoj. 2014. Acontecimiento. Traducción de Raquel Vicedo. Barcelona: Sexto Piso

Notas

${ }^{1}$ Vídeo de Vimeo "At one's fingertips" (2011), de David Sanz Kirbis. https://vimeo. $\underline{\mathrm{com} / 36708258}$ 